\title{
On the extremal values of the ratios of number of paths
}

\author{
Damir Vukičević \\ Department of Mathematics, Faculty of Mathematics and Natural Sciences \\ University of Split, Nikole Tesle 12, HR-21000 Split, Croatia \\ Tomaž Pisanski \\ Department of Theoretical Computer Science, IMFM and \\ University of Ljubljana, Jadranska 19, 1000 Ljubljana, Slovenia
}

Received 12 July 2008, accepted 1 January 2010, published online 26 December 2010

\begin{abstract}
In this paper, we analyze the ratios of the numbers of paths $p_{i}(G)$ and $p_{j}(G)$ of different length in graph $G$. Namely, we are interested in the extremal values of these ratios for acyclic and cyclic graphs with given maximal degree. The values of infinum and supremum for graphs with given maximal degree are obtained. Also, the infinum of these ratios for trees with given maximal degree are obtained. Suprema for trees of given maximal degree are given when ratios of paths of length 1 and 2 are observed, and when ratios of paths of lengths 1 and 3 are observed. As the main result, a linear algorithm (in terms of maximal degree) for finding suprema of the ratios of the numbers of paths of length 2 and 3 for trees with given maximal degree is presented.
\end{abstract}

Keywords: Extremal graph, path, push to leaves.

Math. Subj. Class.: 05C35, 05C38

\section{Introduction}

In this paper, we analyze the possible values of the ratio of the numbers of the paths of lengths $i$ and $j, i>j$. Namely we are interested in the extremal values [2] of the ratio $\frac{p_{i}(G)}{p_{j}(G)}$ where $p_{i}(G)$ and $p_{j}(G)$ are the numbers of (unoriented) paths of length $i$ and $j$, respectively. We restrict ourselves to the simple graphs and henceforth the term graph shall imply simple graph.

E-mail addresses: vukicevi@pmfst.hr (Damir Vukičević), tomaz.pisanski@fmf.uni-lj.si (Tomaž Pisanski) 
Denote by $\mathcal{T}(\Delta, j)$ the family of all trees with maximum degree $\Delta$ that contain at least one path of length $j$ and by $\mathcal{G}(\Delta, j)$ family of connected graphs of maximum degree $\Delta$ that contain at least one path of length $j$. We define functions: $\phi_{i j}^{G}, \Phi_{i j}^{G}, \phi_{i j}^{T}, \Phi_{i j}^{T}: N \backslash\{1\} \rightarrow R$ by

$$
\begin{aligned}
\phi_{i j}^{G}(\Delta) & =\inf _{G \in \mathcal{G}(\Delta, j)} \frac{p_{i}(G)}{p_{j}(G)} ; \\
\phi_{i j}^{T}(\Delta) & =\inf _{T \in \mathcal{T}(\Delta, j)} \frac{p_{i}(T)}{p_{j}(T)} ; \\
\Phi_{i j}^{G}(\Delta) & =\sup _{G \in \mathcal{G}(\Delta, j)} \frac{p_{i}(G)}{p_{j}(G)} ; \\
\Phi_{i j}^{T}(\Delta) & =\sup _{T \in \mathcal{T}(\Delta, j)} \frac{p_{i}(T)}{p_{j}(T)} .
\end{aligned}
$$

for all $i, j \in N, i>j$.

Remark 1.1. Removing the requirement of connectivity in the definition of $\mathcal{G}(\Delta, j)$, then $\phi_{i j}^{G}(\Delta)=0$, since

$$
\lim _{x \rightarrow \infty} \frac{p_{i}\left(G+x \cdot P_{j+1}\right)}{p_{j}\left(G+x \cdot P_{j+1}\right)}=\lim _{x \rightarrow \infty} \frac{p_{i}(G)}{p_{j}(G)+x}=0 .
$$

for all $G \in \mathcal{G}(\Delta, j)$ where $G+x \cdot P_{j+1}$ is the disjoint union of $G$ and $x$ paths of length $j$. On the other hand if $G$ is a graph with connected components $G_{1}, \ldots, G_{k}$ we have

$$
\begin{array}{r}
\frac{p_{i}(G)}{p_{j}(G)} \leq \max \left\{\frac{p_{i}\left(G_{1}\right)}{p_{j}\left(G_{1}\right)}, \ldots, \frac{p_{i}\left(G_{k}\right)}{p_{j}\left(G_{k}\right)}\right\} \leq \max \left\{\Phi_{i j}^{G}(1), \Phi_{i j}^{G}(2), \ldots, \Phi_{i j}^{G}(\Delta)\right\} \\
=\{\text { see remark } 2.14\} \leq \Phi_{i j}^{G}(\Delta)
\end{array}
$$

hence $\Phi_{i j}^{G}$ does not change whether we require connectivity or not. Therefore, we can restrict ourselves to connected graphs. From now on, we use the term graph to imply simple connected graph.

Finding an extremal value of, not a single invariant, but an arithmetic operation on two invariants is not a new concept. It is the concept at the core of AutoGraphX software [1]. Here, we choose paths. They are well known mathematical objects. But, they are also important descriptors in chemistry, especially paths of length two and three. Their numbers are closely related to the Zagreb indices $M_{1}$ and $M_{2}$ which are very well known in chemistry (see $[3,6,8]$ and references within), defined by:

$$
\begin{aligned}
& M_{1}(G)=\sum_{v \in V(G)} d_{G}(v)^{2} ; \\
& M_{2}(G)=\sum_{u v \in E(G)} d_{G}(u) \cdot d_{G}(v) ;
\end{aligned}
$$

where $d_{G}(u)$ is the degree of a vertex $u$ in the graph $G, V(G)$ is the set of vertices of $G$, and $E(G)$ is the set of edges of $G$. It can be shown that:

$$
p_{2}(G)=\frac{1}{2} M_{1}(G)-e(G),
$$


where $e(G)$ is the number of edges of graph $G$ and that

$$
p_{3}(G)=M_{2}(G)-M_{1}(G)+e(G)
$$

for all triangle-free graphs. Comparisons of the Zagreb indices have been extensively studied $[4,9,10,11]$. Besides this, path numbers are themselves interesting molecular descriptors. References about the use of path numbers in defining molecular descriptors and their applications in chemistry can be found in [7].

\section{Results for the infimum}

Denote by $G(\Delta, x)$ the graph presented in the following figure:

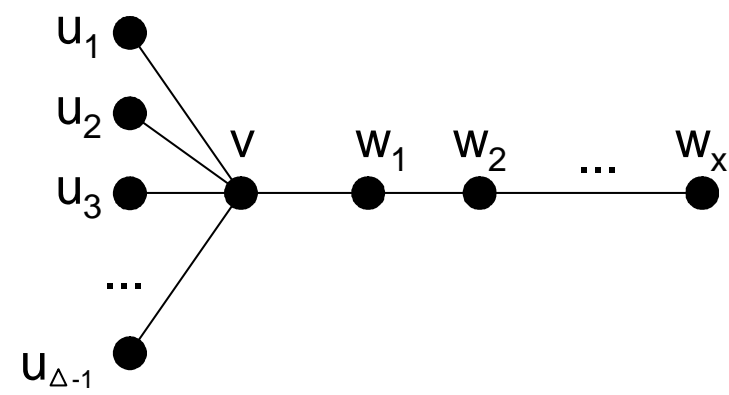

Let us prove:

Proposition 2.1. It holds that $\phi_{i j}^{G}(\Delta)=0$ and $\phi_{i j}^{T}(\Delta)=0$ for all $j<i, i \geq 3$ and $\Delta \in N \backslash\{1\}$.

Proof. Just note that $p_{i}(G(\Delta, j-1))=0$ and that $p_{j}(G(\Delta, j-1))>0$.

Proposition 2.2. It holds that

$$
\phi_{21}^{G}(2)=\phi_{21}^{T}(2)=\frac{1}{2} \text { and } \phi_{21}^{G}(\Delta)=\phi_{21}^{T}(\Delta)=1
$$

for all $\Delta \in N \backslash\{1,2\}$.

Proof. The only graphs with $\Delta=2$ are the path $P_{n}$ on $n$ vertices and the cycle $C_{n}$ on $n$ vertices, where $n \geq 3$. It holds that:

$$
\frac{p_{2}\left(C_{n}\right)}{p_{1}\left(C_{n}\right)}=\frac{n}{n}=1,
$$

and

$$
\frac{p_{2}\left(P_{n}\right)}{p_{1}\left(P_{n}\right)}=\frac{n-2}{n-1},
$$

hence

$$
\phi_{21}^{G}(2)=\phi_{21}^{T}(2)=\inf _{n \geq 3} \frac{n-2}{n-1}=\frac{1}{2} .
$$


Note that

$$
\frac{p_{2}(G(\Delta, x))}{p_{1}(G(\Delta, x))}=\frac{\left(\begin{array}{c}
\Delta \\
2
\end{array}\right)+(x-1) \cdot\left(\begin{array}{l}
2 \\
2
\end{array}\right)}{\Delta-1+x},
$$

hence

$$
\phi_{21}^{G}(\Delta) \leq \phi_{21}^{T}(\Delta) \leq \lim _{x \rightarrow \infty} \frac{\left(\begin{array}{l}
\Delta \\
2
\end{array}\right)+(x-1) \cdot\left(\begin{array}{l}
2 \\
2
\end{array}\right)}{\Delta-1+x}=1 .
$$

In order to prove that

$$
\phi_{21}^{T}(\Delta) \geq \phi_{21}^{G}(\Delta) \geq 1
$$

for every $\Delta \geq 3$, it is sufficient to show that

$$
p_{2}(G)-p_{1}(G) \geq 0 \text { for each } G \text { with } \Delta \geq 3
$$

Denote by $n_{i}$ the number of vertices of degree $i$ in graph $G$ with maximum degree at least 3 , and by $v(G)$ the (total) number of its vertices. We have

$$
\begin{aligned}
e(G) & \geq v(G)-1 \\
\frac{\sum_{i=1}^{\Delta} i \cdot n_{i}}{2} & \geq \sum_{i=1}^{\Delta} n_{i}-1 \\
n_{1} & \leq \sum_{i=3}^{\Delta}(i-2) n_{i}+2 \\
n_{1} & \leq \sum_{i=3}^{\Delta} i \cdot n_{i}
\end{aligned}
$$

hence

$$
\begin{aligned}
p_{2}(G)-p_{1}(G) & =\sum_{i=1}^{\Delta}\left(\begin{array}{l}
i \\
2
\end{array}\right) n_{i}-\frac{\sum_{i=1}^{\Delta} i \cdot n_{i}}{2}=\frac{1}{2} \sum_{i=1}^{\Delta}\left(i^{2}-2 i\right) n_{i}= \\
& =\frac{1}{2}\left[\sum_{i=3}^{\Delta}\left(i^{2}-2 i\right) n_{i}-n_{1}\right] \geq \frac{1}{2}\left[\sum_{i=3}^{\Delta}\left(i^{2}-2 i\right) n_{i}-\sum_{i=3}^{\Delta} i \cdot n_{i}\right]= \\
& =\frac{1}{2} \sum_{i=1}^{\Delta}\left(i^{2}-3 i\right) n_{i} \geq 0
\end{aligned}
$$

Let $P$ be any path. We say that $P^{\prime}$ is an end-subpath of $P$ if it is a subpath of $P$ and if it contains an end-vertex of $P$.

Proposition 2.3. Let $\Delta \geq 2$ and $i>j$. Then,

$$
\Phi_{i j}^{G}(\Delta)=(\Delta-1)^{i-j}
$$

Proof. Let $G$ be any graph. Note that each path of length $i$ contains two paths of length $j$ as end-subpaths. On the other hand any path of length $j$ can be end-subpath of at most 
$2 \cdot(\Delta-1)^{i-j}$ paths of length $i$, because we have 2 choices for the direction of the extension and at most $\Delta-1$ choices for adding each subsequent vertex. Therefore,

$$
p_{j}(G) \leq \frac{2 \cdot(\Delta-1)^{i-j}}{2} \leq(\Delta-1)^{i-j},
$$

hence

$$
\Phi_{i j}^{G}(\Delta) \leq(\Delta-1)^{i-j} .
$$

Now, let $G$ be a $\Delta$-uniform graph (i.e. a graph in which all vertices have degree $\Delta$ ) without a cycle of length less then $\Delta+1$. The existence of such graph follows from the results of paper [5]. Then

$$
\frac{p_{i}(G)}{p_{j}(G)}=\frac{\frac{1}{2} \cdot v(G) \cdot \Delta \cdot(\Delta-1)^{i-1}}{\frac{1}{2} \cdot v(G) \cdot \Delta \cdot(\Delta-1)^{j-1}}=(\Delta-1)^{i-j}
$$

This proves the Theorem.

Determining the functions $\Phi_{i j}^{T}$ is a much harder problem. Here, we restrict ourselves to the cases $i, j \leq 3$, i.e. to analyses of the functions $\Phi_{21}^{T}, \Phi_{31}^{T}$ and $\Phi_{32}^{T}$. First, let us determine $\Phi_{21}^{T}$ :

Proposition 2.4. Let $\Delta \geq 2$. Then

$$
\Phi_{21}^{T}(\Delta)=\frac{\Delta}{2}
$$

Proof. Let $T$ be any tree and $n_{i}$ number of the vertices of degree $i, i=1, \ldots, \Delta$. It holds that:

$$
\frac{p_{2}(T)}{p_{1}(T)}=\frac{\sum_{i=1}^{\Delta}\left(\begin{array}{c}
i \\
2
\end{array}\right) n_{i}}{\frac{1}{2} \sum_{i=1}^{\Delta} i \cdot n_{i}}=\frac{\sum_{i=1}^{\Delta}\left(i^{2}-i\right) n_{i}}{\sum_{i=1}^{\Delta} i \cdot n_{i}}=(*) .
$$

From $e(T)=v(T)-1$, it follows that

$$
\begin{aligned}
\frac{\sum_{i=1}^{\Delta} i \cdot n_{i}}{2} & =\sum_{i=1}^{\Delta} n_{i}-1 \\
n_{1}-2 & =\sum_{i=3}^{\Delta}(i-2) n_{i} .
\end{aligned}
$$

Hence

$$
\begin{aligned}
(*) & =\frac{n_{1} \cdot\left(1^{2}-1\right)+n_{2} \cdot\left(2^{2}-2\right)+\sum_{i=3}^{\Delta}\left(i^{2}-i\right) n_{i}}{2 \cdot 1+\left(n_{1}-2\right) \cdot 1+n_{2} \cdot 2+\sum_{i=3}^{\Delta} i \cdot n_{i}} \\
& =\frac{0+2 n_{2}+\sum_{i=3}^{\Delta}\left(i^{2}-i\right) n_{i}}{2+2 n_{2}+\sum_{i=3}^{\Delta}(2 i-2) \cdot n_{i}} \\
& \leq \max \left\{\left\{\frac{0}{2}, \frac{2}{2}\right\} \cup\left\{\frac{i^{2}-i}{2 i-2}: i=3, . ., \Delta\right\}\right\}=\frac{\Delta}{2} .
\end{aligned}
$$


Therefore $\Phi_{21}^{T}(\Delta) \leq \frac{\Delta}{2}$. Let $T(\Delta, k)$ be a tree with the distinguished vertex $v$ such that all vertices have degree either $\Delta$ or 1 , and all leaves are at distance $k$ from the root. Then

$$
\begin{aligned}
\lim _{k \rightarrow \infty} \frac{p_{2}(T(\Delta, k))}{p_{1}(T(\Delta, k))} & =\lim _{k \rightarrow \infty} \frac{\left(1+\Delta \cdot \sum_{i=0}^{k-2}(\Delta-1)^{i}\right) \cdot\left(\begin{array}{l}
\Delta \\
2
\end{array}\right)}{\left[\left(1+\Delta \cdot \sum_{i=0}^{k-2}(\Delta-1)^{i}\right) \cdot \Delta+\Delta \cdot(\Delta-1)^{k-1}\right] / 2} \\
& =\lim _{k \rightarrow \infty} \frac{\Delta \cdot \frac{(\Delta-1)^{k-1}-1}{\Delta-2} \cdot \Delta \cdot(\Delta-1)}{\Delta \cdot \frac{(\Delta-1)^{k-1}-1}{\Delta-2} \cdot \Delta+\Delta \cdot(\Delta-1)^{k-1}}= \\
& =\lim _{k \rightarrow \infty} \frac{\Delta \cdot(\Delta-1)^{k-1} \cdot \Delta \cdot(\Delta-1)}{\Delta \cdot(\Delta-1)^{k-1} \cdot \Delta+\Delta \cdot(\Delta-1)^{k-1} \cdot(\Delta-2)}= \\
& =\frac{\Delta \cdot(\Delta-1)}{\Delta+(\Delta-2)}=\frac{\Delta^{2}-\Delta}{2 \Delta-2}=\frac{\Delta}{2} .
\end{aligned}
$$

Hence, $\Phi_{21}^{T}(\Delta) \geq \frac{\Delta}{2}$.

Now, we shall need the concept of "pushed to leaves" function. Let $T$ be a rooted tree with root $r$ and let $\rho: E(T) \rightarrow R$ be any function. The "pushed to leaves" function $\rho^{r}: L(T) \rightarrow R(L(T)$ from the set of leaves to the set of real numbers is defined by pushing the weight of the edges to the leaves in the following way: Let $l$ be any leaf and $r v_{1} \ldots v_{k} l$ a path from $r$ to $l$. Then

$$
\begin{aligned}
& \rho^{r}(l)=\frac{\rho\left(r v_{1}\right)}{\left(d\left(v_{1}\right)-1\right)\left(d\left(v_{2}\right)-1\right) \ldots\left(d\left(v_{k}\right)-1\right)}+\frac{\rho\left(v_{1} v_{2}\right)}{\left(d\left(v_{2}\right)-1\right) \ldots\left(d\left(v_{k}\right)-1\right)}+ \\
& +\cdots+\frac{\rho\left(v_{k-1} v_{k}\right)}{d\left(v_{k}\right)-1}+\rho\left(v_{k} l\right) .
\end{aligned}
$$

An example of how the weight of a single edge is pushed to the leaves is presented in the following figure:

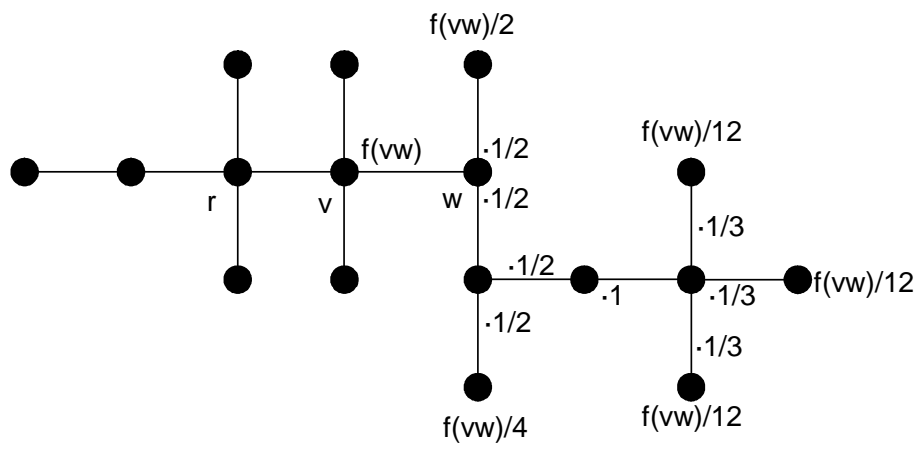

It can easily be seen that $\sum_{u v \in E(T)} \rho(u v)=\sum_{v \in L(T)} \rho^{r}(v)$. Now, let us prove:

Proposition 2.5. Let $\Delta \geq 2$. Then

$$
\Phi_{31}^{T}(\Delta)=\Delta-1
$$


Proof. First, let us prove that $\Phi_{31}^{T}(\Delta)=\Delta-1$. Let $T$ be any tree. Note that the number of paths of length 3 having a middle edge $u v$ is $(d(u)-1) \cdot(d(v)-1)$. Hence

$$
\begin{aligned}
& p_{3}(T)=\sum_{u v \in E(T)}(d(u)-1) \cdot(d(v)-1) ; \\
& p_{1}(T)=\sum_{u v \in E(T)} 1
\end{aligned}
$$

Choose any vertex $r \in V(T) \backslash L(T)$. Since $p_{3}$ and $p_{2}$ are expressed as the sum of the contributions of edge-weights, the functions $p_{1}^{r}$ and $p_{2}^{r}$ can be defined and we have:

$$
\frac{p_{3}(T)}{p_{1}(T)}=\frac{p_{3}^{r}(T)}{p_{1}^{r}(T)}=\frac{\sum_{v \in L(T)} p_{3}^{r}(v)}{\sum_{v \in L(T)} p_{1}^{r}(v)} \leq \max _{v \in L(T)} \frac{p_{3}^{r}(v)}{p_{1}^{r}(v)}
$$

Let $v v_{1} v_{2} \ldots v_{k-1} v_{k}\left(v_{k}=r\right)$ be a path from $v$ to $r$ and denote $d_{i}=d\left(v_{i}\right)$ We have:

$$
\begin{aligned}
& \max _{v \in L(T)} \frac{p_{3}^{r}(v)}{p_{2}^{r}(v)}= \\
& =\frac{\left[\begin{array}{c}
(1-1) \cdot\left(d_{1}-1\right)+\frac{\left(d_{1}-1\right) \cdot\left(d_{2}-1\right)}{d_{1}-1}+\frac{\left(d_{2}-1\right) \cdot\left(d_{3}-1\right)}{\left(d_{1}-1\right) \cdot\left(d_{2}-1\right)}+ \\
+\cdots+\frac{\left(d_{k-1}-1\right) \cdot\left(d_{k}-1\right)}{\left(d_{1}-1\right) \cdot\left(d_{2}-1\right) \cdots \cdot\left(d_{k-2}-1\right) \cdot\left(d_{k-1}-1\right)}
\end{array}\right]}{\left[\begin{array}{c}
1+\frac{1}{d_{1}-1}+\frac{1}{\left(d_{1}-1\right) \cdot\left(d_{2}-1\right)}+ \\
+\cdots+\frac{1}{\left(d_{1}-1\right) \cdot\left(d_{2}-1\right) \cdots \cdot\left(d_{k-2}-1\right)}+\frac{1}{\left(d_{1}-1\right) \cdot\left(d_{2}-1\right) \cdots \cdot\left(d_{k-2}-1\right) \cdot\left(d_{k-1}-1\right)}
\end{array}\right]} \\
& \leq \frac{\left(d_{2}-1\right)+\frac{d_{3}-1}{\left(d_{1}-1\right)}+\cdots+\frac{d_{k}-1}{\left(d_{1}-1\right) \cdot\left(d_{2}-1\right) \cdots \cdot\left(d_{k-2}-1\right)}}{\left[\begin{array}{c}
1+\frac{1}{d_{1}-1}+\frac{1}{\left(d_{1}-1\right) \cdot\left(d_{2}-1\right)}+ \\
+\cdots+\frac{1}{\left(d_{1}-1\right) \cdot\left(d_{2}-1\right) \cdots \cdot\left(d_{k-2}-1\right)}+\frac{1}{\left(d_{1}-1\right) \cdot\left(d_{2}-1\right) \cdots \cdot\left(d_{k-2}-1\right) \cdot\left(d_{k-1}-1\right)}
\end{array}\right]} \text {. } \\
& \leq \frac{\Delta-1+\frac{\Delta-1}{\left(d_{1}-1\right)}+\cdots+\frac{\Delta-1}{\left(d_{1}-1\right) \cdot\left(d_{2}-1\right) \cdots \cdot\left(d_{k-2}-1\right)}}{\left[\begin{array}{c}
1+\frac{1}{d_{1}-1}+\frac{1}{\left(d_{1}-1\right) \cdot\left(d_{2}-1\right)}+ \\
+\cdots+\frac{1}{\left(d_{1}-1\right) \cdot\left(d_{2}-1\right) \cdots \cdot\left(d_{k-2}-1\right) \cdot\left(d_{k-1}-1\right)}
\end{array}\right]} \\
& \leq \Delta-1 \text {. }
\end{aligned}
$$

Hence, $\Phi_{31}^{T}(\Delta) \leq \Delta-1$. Let $T(\Delta, k)$ be defined as above. We have:

$$
\begin{aligned}
\lim _{k \rightarrow \infty} \frac{p_{3}(T(\Delta, k))}{p_{1}(T(\Delta, k))}= \\
\quad=\lim _{k \rightarrow \infty} \frac{\left(\Delta \cdot \sum_{i=0}^{k-2}(\Delta-1)^{i}\right) \cdot(\Delta-1)^{2}+\left(\Delta \cdot(\Delta-1)^{k-1}\right) \cdot(\Delta-1) \cdot(1-1)}{\left(\Delta \cdot \sum_{i=0}^{k-1}(\Delta-1)^{i}\right)} \\
\quad=\lim _{k \rightarrow \infty} \frac{\Delta \cdot \frac{(\Delta-1)^{k-1}-1}{\Delta-1-1} \cdot(\Delta-1)^{2}}{\Delta \cdot \frac{(\Delta-1)^{k}-1}{\Delta-1-1}}=\lim _{k \rightarrow \infty} \frac{(\Delta-1)^{k-1} \cdot(\Delta-1)^{2}}{(\Delta-1)^{k}}=\Delta-1
\end{aligned}
$$

Hence, $\Phi_{31}^{T}(\Delta) \geq \Delta-1$. 
Determining of $\Phi_{32}^{T}$ is much more complex problem. Let us start with the simplest case: Proposition 2.6. $\Phi_{32}^{T}(2)=1$.

Proof. The tree with maximum degree 2 is a path. Denote by $P_{n}$ the path on $n$ vertices. It holds that $\frac{p_{3}\left(P_{n}\right)}{p_{2}\left(P_{n}\right)}=\frac{n-3}{n-2}$ and therefore

$$
\sup _{T \in \mathcal{T}(2,2)} \frac{p_{3}(T)}{p_{2}(T)}=\sup _{n \geq 3} \frac{n-3}{n-2}=1 .
$$

Denote

$$
f\left(x_{1}, x_{2}, \ldots, x_{k}\right)=\frac{\frac{x_{1} x_{2}}{x_{1}}+\frac{x_{2} x_{3}}{x_{1} x_{2}}+\cdots+\frac{x_{k-1} x_{k}}{x_{1} \ldots x_{k-1}}}{x_{1}+\frac{x_{1}+x_{2}}{x_{1}}+\frac{x_{2}+x_{3}}{x_{1} x_{2}}+\cdots+\frac{x_{k-1}+x_{k}}{x_{1} \ldots x_{k-1}}} .
$$

Note that $f\left(x_{1}, x_{2}, \ldots, x_{k}\right) \leq m / 2$ for all $x_{1}, x_{2}, \ldots, x_{m}$. Hence, it can be defined:

$$
\Gamma(m)=\sup _{k \in N, x_{1}, \ldots, x_{k-1} \in\{1, \ldots, m\}} f\left(x_{1}, x_{2}, \ldots, x_{k-1}, m\right) .
$$

Note that $k=1$ on the right hand-side implies that we observe $f(m)$. Now, we shall prove several auxiliary lemmas:

Lemma 2.7. $\Phi_{32}(\Delta)=2 \cdot \Gamma(\Delta-1)$ for all $\Delta>2$.

Proof. Recall that the number of paths of length 3 with a middle edge $u v$ is $(d(u)-1)$. $(d(v)-1)$ and that the number of paths of length 2 with middle vertex $v$ is $\left(\begin{array}{c}d(v) \\ 2\end{array}\right)$. Hence

$$
\begin{aligned}
p_{3}(T) & =\sum_{u v \in E(T)}(d(u)-1) \cdot(d(v)-1) ; \\
p_{2}(T) & =\sum_{v \in V(T)}\left(\begin{array}{c}
d(v) \\
2
\end{array}\right)=\frac{1}{2} \sum_{v \in V(T)} d(v) \cdot(d(v)-1)= \\
& =\frac{1}{2} \sum_{v \in V(T)} \sum_{u \in V(T): u v \in E(T)}(d(v)-1)=\frac{1}{2} \sum_{u v \in E(T)}[(d(u)-1)+(d(v)-1)] .
\end{aligned}
$$

Choose any vertex $r \in V(T)$ of degree $\Delta$. Since $p_{3}$ and $p_{2}$ are expressed as the sum of the contributions of edge-weights, functions $p_{1}^{r}$ and $p_{2}^{r}$ can be defined and we have:

$$
\frac{p_{3}(T)}{p_{2}(T)}=\frac{p_{3}^{r}(T)}{p_{2}^{r}(T)}=\frac{\sum_{v \in L(T)} p_{3}^{r}(v)}{\sum_{v \in L(T)} p_{2}^{r}(v)} .
$$

First, let us prove that

$$
\Phi_{32}(\Delta) \leq 2 \cdot \Gamma(\Delta-1) .
$$

It is sufficient to prove that for each $T \in \mathcal{T}(\Delta, 2)$ it holds that:

$$
\frac{p_{3}(T)}{p_{2}(T)}=\frac{\sum_{v \in L(T)} p_{3}^{r}(v)}{\sum_{v \in L(T)} p_{2}^{r}(v)} \leq 2 . \sup _{k \in N, x_{1}, \ldots, x_{k-1} \in\{1, \ldots, \Delta-1\}} f\left(x_{1}, x_{2}, \ldots, x_{k-1}, \Delta-1\right) .
$$


Note that

$$
\frac{\sum_{v \in L(T)} p_{3}^{r}(v)}{\sum_{v \in L(T)} p_{2}^{r}(v)} \leq \max _{l \in L(T)} \frac{p_{3}^{r}(l)}{p_{2}^{r}(l)}
$$

Denote by $l$ the leaf for which the observed ratio obtains its maximum and let $l v_{1} v_{2} \ldots$ $v_{q}\left(v_{q}=r\right)$ be a path from $l$ to $r$. Denote $d_{i}=d\left(v_{i}\right)$ We have:

$$
\begin{aligned}
\max _{v \in L(T)} \frac{p_{3}^{p}(v)}{p_{2}^{p}(v)}= \\
=\frac{\left[\begin{array}{c}
(1-1) \cdot\left(d_{1}-1\right)+\frac{\left(d_{2}-1\right)\left(d_{1}-1\right)}{\left(d_{1}-1\right)}+ \\
\left.+\frac{\left(d_{3}-1\right)\left(d_{2}-1\right)}{\left(d_{1}-1\right)\left(d_{2}-1\right)}+\cdots+\frac{\left(d_{q-1}-1\right)\left(d_{q}-1\right)}{\left(d_{1}-1\right)\left(d_{2}-1\right) \cdot\left(d_{q-1}-1\right)}\right]
\end{array}\right]}{\frac{1}{2}\left[\begin{array}{c}
(1-1)+\left(d_{1}-1\right)+\frac{\left(d_{2}-1\right)+\left(d_{1}-1\right)}{\left(d_{1}-1\right)}+ \\
+\frac{\left(d_{3}-1\right)+\left(d_{2}-1\right)}{\left(d_{1}-1\right)\left(d_{2}-1\right)}+\cdots+\frac{\left(d_{q-1}-1\right)+\left(d_{q}-1\right)}{\left(d_{1}-1\right)\left(d_{2}-1\right) \cdot\left(d_{q-1}-1\right)}
\end{array}\right]} \\
=2 \cdot f\left(d_{1}-1, d_{2}-1, \ldots, d_{q}-1\right) \\
\leq 2 \cdot \sup _{k \in N, x_{1}, \ldots, x_{k-1} \in\{1, \ldots, \Delta-1\}} f\left(x_{1}, x_{2}, \ldots, x_{k-1}, \Delta-1\right)=2 \cdot \Gamma(\Delta-1) .
\end{aligned}
$$

Now, let us prove that

$$
\Phi(\Delta) \geq 2 \cdot \Gamma(\Delta-1)
$$

It is sufficient to prove that

$$
\Phi(\Delta) \geq 2 \cdot f\left(x_{1}, x_{2}, \ldots, x_{k-1}, \Delta-1\right)
$$

for each $\left(x_{1}, \ldots, x_{k-1}\right)$ where $x_{i} \in\{1, \ldots, \Delta-1\}, i=1, \ldots, k$. Set $x_{k}=\Delta-1$. Denote by $T\left(x_{1}, \ldots, x_{k}\right)$ a tree such that the following hold:

1) There is a distinguished vertex $r \in V\left(T\left(x_{1}, \ldots, x_{k}\right)\right)$ of degree $x_{k}+1$ such that all leaves are at distance $k$ from $r$

2) Let $l v_{1} \ldots v_{k-1} r$ be a path from any leaf $l$ to $v$. Then, $d\left(v_{i}\right)=x_{i}+1$ for every vertex $v_{i}$.

We have:

$$
\Phi(\Delta) \geq \frac{p_{3}\left(T\left(x_{1}, \ldots, x_{k}\right)\right)}{p_{2}\left(T\left(x_{1}, \ldots, x_{k}\right)\right)}=\frac{\sum_{v \in L\left(T\left(x_{1}, \ldots, x_{k}\right)\right)} p_{3}^{r}(v)}{\sum_{v \in L\left(T\left(x_{1}, \ldots, x_{k}\right)\right)} p_{2}^{r}(v)}=2 \cdot f\left(x_{1}, \ldots, x_{k}\right) .
$$

Lemma 2.8. Let $m \geq 2$, then

$$
\Gamma(m) \leq \frac{m^{3 / 2}}{m+1}
$$


Proof. For each $k \in N$, each $x_{1}, \ldots, x_{k-1} \in\{1, \ldots, m\}, x_{k}=m$, and each $\lambda \in[0,1]$, we have

$$
\begin{aligned}
& \frac{\frac{x_{1} x_{2}}{x_{1}}+\frac{x_{2} x_{3}}{x_{1} x_{2}}+\cdots+\frac{x_{k-1} x_{k}}{x_{1} \ldots x_{k-1}}}{x_{1}+\frac{x_{1}+x_{2}}{x_{1}}+\frac{x_{2}+x_{3}}{x_{1} x_{2}}+\cdots+\frac{x_{k-1}+x_{k}}{x_{1} \ldots x_{k-1}}}= \\
& =\frac{\frac{x_{1} x_{2}}{x_{1}}+\frac{x_{2} x_{3}}{x_{1} x_{2}}+\cdots+\frac{x_{k-1} x_{k}}{x_{1} \ldots x_{k-1}}}{(1-\lambda) x_{1}+\frac{\lambda x_{1}^{2}+x_{1}+(1-\lambda) x_{2}}{x_{1}}+\frac{\lambda x_{2}^{2}+x_{2}+(1-\lambda) x_{3}}{x_{1} x_{2}}+\cdots+\frac{\lambda x_{k-1}^{2}+x_{k-1}+x_{k}}{x_{1} \ldots x_{k-1}}} \\
& =\frac{\left[\begin{array}{c}
\frac{\lambda x_{1}^{2}+x_{1}+(1-\lambda) x_{2}}{x_{1}} \cdot \frac{x_{1} x_{2}}{\lambda x_{1}^{2}+x_{1}+(1-\lambda) x_{2}}+ \\
+\frac{\lambda x_{2}^{2}+x_{2}+(1-\lambda) x_{3}}{x_{1} x_{2}} \cdot \frac{x_{2} x_{3}}{\lambda x_{2}^{2}+x_{2}+(1-\lambda) x_{3}}+\cdots+ \\
\frac{\lambda x_{k-1}^{2}+x_{k-1}+x_{k}}{x_{1} \ldots x_{k-1}} \cdot \frac{x_{k-1} x_{k}}{\lambda x_{k-1}^{2}+x_{k-1}+x_{k}}
\end{array}\right]}{(1-\lambda) x_{1}+\frac{\lambda x_{1}^{2}+x_{1}+(1-\lambda) x_{2}}{x_{1}}+\frac{\lambda x_{2}^{2}+x_{2}+(1-\lambda) x_{3}}{x_{1} x_{2}}+\cdots+\frac{\lambda x_{k-1}^{2}+x_{k-1}+x_{k}}{x_{1} \ldots x_{k-1}}}=(*)
\end{aligned}
$$

Let

$$
S=\inf _{\lambda \in[0,1]} \max _{a, b \in\{1, \ldots, m\}} \frac{a b}{a+\lambda a^{2}+(1-\lambda) b}
$$

We have

$$
(*) \leq \frac{\left[\frac{x_{1}+x_{2}+\lambda x_{2}^{2}}{x_{2} x_{3} \ldots x_{k}} \cdot S+\frac{(1-\lambda) x_{2}+x_{3}+\lambda x_{3}^{2}}{x_{3} \ldots x_{k}} \cdot S+\cdots++\frac{(1-\lambda) x_{k-1}+x_{k}+\lambda x_{k}^{2}}{x_{k}} \cdot S\right]}{\left[\frac{x_{1}+x_{2}+\lambda x_{2}^{2}}{x_{2} x_{3} \ldots x_{k}}+\frac{(1-\lambda) x_{2}+x_{3}+\lambda x_{3}^{2}}{x_{3} \ldots x_{k}}+\cdots+\frac{(1-\lambda) x_{k-1}+x_{k}+\lambda x_{k}^{2}}{x_{k}}\right]} \leq S
$$

Let us calculate the upper bound of $\max _{a, b \in\{1, \ldots, m\}} \frac{a b}{a+\lambda a^{2}+(1-\lambda) b}$. We have

$$
\begin{aligned}
\max _{a, b \in\{1, \ldots, m\}} & \frac{a b}{a+\lambda a^{2}+(1-\lambda) b}= \\
& =\max _{a, b \in\{1, \ldots, m\}} \frac{a b}{a^{2} \cdot\left(\lambda+\frac{1}{a}\right)+(1-\lambda) b} \\
& \leq \max _{a, b \in\{1, \ldots, m\}} \frac{a b}{2 \cdot \sqrt{\left(\lambda+\frac{1}{a}\right) a^{2} \cdot b(1-\lambda)}} \\
& =\max _{a, b \in\{1, \ldots, m\}} \frac{\sqrt{b}}{2 \cdot \sqrt{(1-\lambda)\left(\lambda+\frac{1}{a}\right)}} \\
& \leq\{\text { increasing in } a \text { and } b\} \leq \frac{\sqrt{m}}{2 \cdot \sqrt{(1-\lambda)\left(\lambda+\frac{1}{m}\right)}}
\end{aligned}
$$

Using simple analytical calculation to maximize $(1-\lambda)\left(\lambda+\frac{1}{m}\right)$ for $\lambda \in[0,1]$, we obtain

$$
S=\inf _{\lambda \in[0,1]} \frac{\sqrt{m}}{2 \cdot \sqrt{(1-\lambda)\left(\lambda+\frac{1}{m}\right)}}=\frac{m^{3 / 2}}{1+m} .
$$

which proves the claim. 
Denote

$$
\begin{aligned}
& X(m)=\left\{\left(x_{1}, m, m\right): x_{1} \in\{1, \ldots, m\}\right\} \cup \\
& \cup\left\{\left(x_{1}, x_{2}, m\right): x_{1} \in\left\{1, \ldots,\left\lfloor\frac{m^{3 / 2}}{m+1}\right\rfloor\right\}, x_{2} \in\{1, \ldots, m\}\right\} \cup \\
&\left.\cup\left\{\left(x_{1}, x_{2}, x_{3}\right): x_{1}, x_{2} \in\left\{1, \ldots, \mid \frac{m^{3 / 2}}{m+1}\right\rfloor\right\}, x_{3} \in\{1, \ldots, m\}\right\} \\
& g_{m}\left(x_{1}, x_{2}, x_{3}\right)= \frac{\frac{x_{1} x_{2}}{x_{1}}+\frac{x_{2} x_{3}}{x_{1} x_{2}}+\frac{x_{3} m}{x_{1} x_{2} x_{3}}+\frac{1}{x_{1} x_{2} x_{3}} \cdot \frac{m^{2}}{m-1}}{x_{1}+\frac{x_{1}+x_{2}}{x_{1}}+\frac{x_{2}+x_{3}}{x_{1} x_{2}}+\frac{x_{3}+m}{x_{1} x_{2} x_{3}}+\frac{1}{x_{1} x_{2} x_{3}} \cdot \frac{2 m}{m-1}}
\end{aligned}
$$

and

$$
\Psi(m)=\max _{\left(x_{1}, x_{2}, x_{3}\right) \in X(m)} g_{m}\left(x_{1}, x_{2}, x_{3}\right)
$$

Let us prove:

Lemma 2.9. Let $m \geq 2$, then

$$
\frac{m}{m+1} \leq \Psi(m) \leq \frac{m^{3 / 2}}{m+1} \leq \frac{m}{2} .
$$

Proof. First, let us prove that $\Gamma(m) \geq \frac{m}{m+1}$. It is sufficient to prove that

$$
g(1, m, m) \geq \frac{m}{m+1},
$$

i.e. that

$$
\begin{aligned}
\frac{2 m+1+\frac{1}{m-1}}{4+m+\frac{2}{m}+\frac{2}{m(m-1)}} & \geq \frac{m}{m+1} \\
2 m^{2}+3 m+1+\frac{m+1}{m-1} & \geq m^{2}+4 m+2+\frac{2}{m-1} \\
m^{2} & \geq m
\end{aligned}
$$

which is obviously true.

Simple calculation shows that

$$
\frac{m^{3 / 2}}{m+1} \leq \frac{m}{2}
$$

Now, let us prove that $\Gamma(m) \leq \frac{m^{3 / 2}}{m+1}$. It is sufficient to prove that for each

$$
\left(x_{1}, x_{2}, x_{3}\right) \in X
$$

it holds that:

$$
g_{m}\left(x_{1}, x_{2}, x_{3}\right) \leq \frac{m^{3 / 2}}{m+1}
$$

Note that

$$
\lim _{n \rightarrow \infty} f(x_{1}, x_{2}, x_{3}, \underbrace{m, \ldots, m}_{n \text {-times }})=g_{m}\left(x_{1}, x_{2}, x_{3}\right) .
$$


Hence,

$$
g_{m}\left(x_{1}, x_{2}, x_{3}\right) \leq \Gamma(m) \leq \frac{m^{3 / 2}}{m+1}
$$

This proves the Lemma.

Let us prove:

Lemma 2.10. Let $k$ and $m \geq 2$ be positive integers and $r$ real number such that $\frac{m}{m+1} \leq$ $r \leq \frac{m}{2}$, and $k \geq 2$. Then

$$
\begin{aligned}
& \max _{t_{1}, t_{2}, \ldots, t_{k} \in\{1, \ldots, m\}} \frac{t_{1} t_{2}-r t_{1}-r t_{2}}{t_{1}}+\frac{t_{2} t_{3}-r t_{2}-r t_{3}}{t_{1} t_{2}}+\cdots+\frac{t_{k-1} t_{k}-r t_{k-1}-r t_{k}}{t_{1} t_{2} t_{3} \ldots t_{k-1}} \\
& \leq(m-2 r) \cdot \frac{1-\frac{1}{m^{k}}}{1-\frac{1}{m}}
\end{aligned}
$$

Proof. We prove the claim by induction on $k$. First suppose that $k=2$. It is sufficient to prove that

$$
\frac{t_{1} t_{2}-r t_{1}-r t_{2}}{t_{1}} \leq m-2 r
$$

Note that $m-2 r \geq 0$. If $t_{1} \leq r$ then $t_{1} t_{2}-r t_{1}-r t_{2}$ is negative and the claim holds. If $t_{1}>r$, the left hand-side is increasing in $t_{2}$, hence

$$
\frac{t_{1} t_{2}-r t_{1}-r t_{2}}{t_{1}} \leq \frac{t_{1} m-r t_{1}-r m}{t_{1}}=m-r-\frac{r m}{t_{1}} \leq\left\{\text { increasing in } t_{1}\right\} \leq m-2 r .
$$

Now, suppose that $k>2$ and that claim holds for smaller values of $k$. We have:

$$
\begin{aligned}
& \frac{t_{1} t_{2}-r t_{1}-r t_{2}}{t_{1}}+\frac{t_{1} t_{2}-r t_{1}-r t_{2}}{t_{1} t_{2}}+\frac{t_{2} t_{3}-r t_{2}-r t_{3}}{t_{1} t_{2} t_{3}}+\cdots+\frac{t_{k-1} t_{k}-r t_{k-1}-r t_{k}}{t_{1} t_{2} t_{3} \ldots t_{k-1}} \\
& =\frac{t_{1} t_{2}-r t_{1}-r t_{2}}{t_{1}}+ \\
& \quad \frac{1}{t_{1}} \cdot\left(\frac{t_{2} t_{3}-r t_{2}-r t_{3}}{t_{2}}+\frac{t_{3} t_{4}-r t_{3}-r t_{4}}{t_{2} t_{3}}+\cdots+\frac{t_{k-1} t_{k}-r t_{k-1}-r t_{k}}{t_{2} t_{3} \ldots t_{k-1}}\right)
\end{aligned}
$$

$\leq\{$ by the inductive hypothesis $\}$

$$
=\frac{t_{1} t_{2}-r t_{1}-r t_{2}}{t_{1}}+\frac{1}{t_{1}}(m-2 r) \cdot \frac{1-\frac{1}{m^{k-1}}}{1-\frac{1}{m}}
$$

If $t_{1} \leq r$ then

$$
\begin{aligned}
\frac{t_{1} t_{2}-r t_{1}-r t_{2}}{t_{1}}+\frac{1}{t_{1}}(m-2 r) \cdot \frac{1-\frac{1}{m^{k-1}}}{1-\frac{1}{m}} & \leq \frac{1}{t_{1}}(m-2 r) \cdot \frac{1-\frac{1}{m^{k-1}}}{1-\frac{1}{m}} \\
& \leq(m-2 r) \cdot \frac{1-\frac{1}{m^{k-1}}}{1-\frac{1}{m}} \\
& \leq(m-2 r) \cdot \frac{1-\frac{1}{m^{k}}}{1-\frac{1}{m}}
\end{aligned}
$$


Otherwise,

$$
\begin{aligned}
\frac{t_{1} t_{2}-r t_{1}-r t_{2}}{t_{1}}+ & \frac{1}{t_{1}}(m-2 r) \cdot \frac{1-\frac{1}{m^{k-1}}}{1-\frac{1}{m}} \\
& \leq\left\{\text { increasing in } t_{2}\right\} \\
& \leq \frac{m t_{1}-r t_{1}-r m}{t_{1}}+\frac{1}{t_{1}}(m-2 r) \cdot \frac{1-\frac{1}{m^{k-1}}}{1-\frac{1}{m}} \\
& =m-r+\frac{1}{t_{1}} \cdot\left[(m-2 r) \cdot \frac{1-\frac{1}{m^{k-1}}}{1-\frac{1}{m}}-r m\right]=(*)
\end{aligned}
$$

In order to prove that $(*)$ is increasing in $t_{1}$, we need to prove that

$$
(m-2 r) \cdot \frac{1-\frac{1}{m^{k-1}}}{1-\frac{1}{m}}-r m \leq 0 .
$$

It is sufficient to prove that

$$
\frac{m-2 r}{1-\frac{1}{m}} \leq r m
$$

but this is equivalent to

$$
\begin{aligned}
m & \leq r m-r+2 r \\
r & \geq \frac{m}{m+1}
\end{aligned}
$$

Therefore, $(*)$ is increasing in $t_{1}$, and

$$
\begin{aligned}
(*) & \leq m-r+\frac{1}{m} \cdot\left[(m-2 r) \cdot \frac{1-\frac{1}{m^{k-1}}}{1-\frac{1}{m}}-r m\right] \\
& =(m-2 r) \cdot\left(1+\frac{1}{m} \cdot \frac{1-\frac{1}{m^{k-1}}}{1-\frac{1}{m}}\right)=(m-2 r) \cdot \frac{1-\frac{1}{m^{k}}}{1-\frac{1}{m}}
\end{aligned}
$$

which proves the Lemma.

Now, let us prove the key Lemma.

Lemma 2.11. Let $m \geq 2$, then

$$
\Gamma(m)=\Psi(m) .
$$

Proof. Denote $r=\Psi(m)$ and denote $\left(y_{1}, y_{2}, y_{3}\right) \in X(m)$ such that $g_{m}\left(y_{1}, y_{2}, y_{3}\right)=r$. We have:

$$
\begin{aligned}
\Gamma(m) & =\sup _{k \in N, x_{1}, \ldots, x_{k} \in\{1,2, \ldots, m\}} f\left(x_{1}, \ldots, x_{k}\right) \geq \lim _{k \rightarrow \infty} f(y_{1}, y_{2}, y_{3}, \underbrace{m, \ldots, m}_{(k-3) \text {-times }}) \\
& =\lim _{k \rightarrow \infty} \frac{\frac{y_{1} y_{2}}{y_{1}}+\frac{y_{2} y_{3}}{y_{1} y_{2}}+\frac{y_{3} m}{y_{1} y_{2} y_{3}}+\frac{m^{2}}{y_{1} y_{2} y_{3}} \cdot\left(\frac{1}{m}+\frac{1}{m^{2}}+\cdots+\frac{1}{m^{k-4}}\right)}{y_{1}+\frac{y_{1}+y_{2}}{y_{1}}+\frac{y_{2}+y_{3}}{y_{1} y_{2}}+\frac{y_{3}+m}{y_{1} y_{2} y_{3}}+\frac{2 m}{y_{1} y_{2} y_{3}} \cdot\left(\frac{1}{m}+\frac{1}{m^{2}}+\cdots+\frac{1}{m^{k-4}}\right)} \\
& =g_{m}\left(y_{1}, y_{2}, y_{3}\right)=r
\end{aligned}
$$


Suppose to the contrary that

$$
\sup _{k \in N, x_{1}, \ldots, x_{k-1} \in\{1,2, \ldots, m\}} f\left(x_{1}, \ldots, x_{k-1}, m\right)>r .
$$

Denote by $S_{1}$ set of finite ordered sequences $\left(x_{1}, \ldots, x_{k-1}, x_{k}\right)$ such that $x_{k}=m$ and

$$
f\left(x_{1}, \ldots, x_{k}\right)>r .
$$

Note that this last relation can be rewritten as

$$
\begin{aligned}
h\left(x_{1}, \ldots, x_{k}\right)=-r x_{1}+\frac{x_{1} x_{2}-r x_{1}-r x_{2}}{x_{1}}+ & \\
& \frac{x_{2} x_{3}-r x_{2}-r x_{3}}{x_{1} x_{2}}+\cdots+\frac{x_{k-1} x_{k}-r x_{k-1}-r x_{k}}{x_{1} \ldots x_{k-1}}>0
\end{aligned}
$$

From (2.1), it follows that $S_{1}$ is a non-empty set. Let $S_{2}$ be the set of sequences in $S_{1}$ which have one of the following two properties:

1) There are no entries from $[r, m\rangle$ and all $m$ s are located at the end of the sequence;

2) There is a single entry from the set $[r, m\rangle$; there is no $m$ before this entry and all entries after this one are equal to $m$.

Let us prove that $S_{3}$ is non-empty. Let $\left(b_{1}, \ldots, b_{k_{2}}\right) \in S_{2}$. Let $i$ be the first entry greater or equal $r$ (note that at least $b_{k_{2}}=m \geq r$ ). If $i=k_{2}$, then $\left(b_{1}, \ldots, b_{k_{2}}\right) \in S_{2}$, hence suppose that $i<k_{2}$. In order to prove that $(b_{1}, \ldots, b_{i}, \underbrace{m, \ldots, m}_{\left(k_{2}-i\right) \text {-times }}) \in S_{2}$, it is sufficient to prove that

$$
h(b_{1}, \ldots, b_{i}, \underbrace{m, \ldots, m}_{\left(k_{2}-i\right) \text {-times }}) \geq h\left(b_{1}, \ldots, b_{k_{2}}\right) \geq 0
$$

i.e. that

$$
h(b_{1}, \ldots, b_{i}, \underbrace{m, \ldots, m}_{\left(k_{2}-i\right) \text {-times }})-h\left(b_{1}, \ldots, b_{k_{2}}\right) \geq 0 \text {. }
$$

We have:

$$
\begin{aligned}
& \left(h(b_{1}, \ldots, b_{i}, \underbrace{m, \ldots, m}_{\left(k_{2}-i\right) \text {-times }})-h\left(b_{1}, \ldots, b_{k_{2}}\right)\right) \cdot b_{1} b_{2} \ldots b_{i}= \\
& =\left(b_{i} m-r m-r b_{i}\right)+\frac{m \cdot m-2 r m}{m}+\frac{m \cdot m-2 r m}{m^{2}}+\cdots+\frac{m \cdot m-2 r m}{m^{k_{2}-i-1}} \\
& \quad-\left(b_{i} b_{i+1}-r b_{i}-r b_{i+1}\right)-\frac{b_{i+1} b_{i+2}-r b_{i+1}-r b_{i+2}}{b_{i+1}}-\ldots \\
& \quad-\frac{b_{k_{2}-1} b_{k_{2}}-r b_{k_{2}-1}-r b_{k_{2}}}{b_{i+1} \ldots b_{k_{2}}}= \\
& =\left[\left(b_{i}-r\right) \cdot\left(m-b_{i+1}\right)\right] \\
& \left.\quad+\left[\begin{array}{c}
\left(\frac{m \cdot m-2 r m}{m}+\frac{m \cdot m-2 r m}{m^{2}}+\cdots+\frac{m \cdot m-2 r m}{m^{k}-i-1}\right)- \\
b_{i+1} b_{i+2}-r b_{i+1}-r b_{i+2} \\
b_{i+1}
\end{array}+\cdots+\frac{b_{k_{2}-1} b_{k_{2}}-r b_{k_{2}}-1-r b_{k_{2}}}{b_{i+1} \ldots b_{k_{2}}}\right)\right] .
\end{aligned}
$$


The first square bracket is non-negative because it is the product of two non-negative numbers. From Lemma 2.9 it follows that $\frac{m}{m+2} \leq r \leq \frac{m}{2}$ and then from Lemma 2.10, it follows that the second square bracket is non-negative, so $S_{2}$ is non-empty. Let $c=\left(c_{1}, \ldots, c_{k_{2}}\right)$ be an element of $S_{2}$ with the following properties:

1) $c$ has the least number of entries smaller then $r$;

2) Among all the elements of $S_{2}$ with the same number of entries smaller then $r, c$ is the shortest sequence.

Note that all these entries are at the beginning of the sequence. Hence, assume that $c_{1}, \ldots, c_{j}$ are smaller then $r$ and $c_{j+1}, \ldots, c_{k_{3}}$ are larger then $r$. Distinguish two cases:

CASE 1: $h\left(c_{1}, \ldots, c_{k_{3}}\right) \geq h\left(c_{1}, c_{2}\right)$

SUBCASE 1.1: $j \geq 3$.

Because of the minimality of $\left(c_{1}, \ldots, c_{k_{3}}\right)$, it follows that $\left(c_{1}, c_{3}, c_{4}, c_{k_{3}}\right) \notin S_{3}$, hence

$$
\begin{aligned}
h\left(c_{1}, \ldots, c_{k_{3}}\right) & >0 \\
h\left(c_{1}, c_{3}, c_{4}, \ldots, c_{k_{3}}\right) & <0
\end{aligned}
$$

Therefore

$$
h\left(c_{1}, \ldots, c_{k_{3}}\right)>h\left(c_{1}, c_{3}, c_{4}, \ldots, c_{k_{3}}\right) .
$$

We have:

$$
\begin{aligned}
& h\left(c_{1}, \ldots, c_{k_{3}}\right) \leq h\left(c_{1}, \ldots, c_{k_{3}}\right)+\left(c_{2}-1\right) \cdot\left(h\left(c_{1}, \ldots, c_{k_{3}}\right)-h\left(c_{1}, c_{2}\right)\right) \\
& \leq-r c_{1}+\frac{c_{1} c_{2}-r c_{1}-r c_{2}}{c_{1}}+\frac{c_{2} c_{3}-r c_{2}-r c_{3}}{c_{1}}+\frac{c_{3} c_{4}-r c_{3}-r c_{4}}{c_{1} c_{3}}+\ldots \\
& \quad+\frac{c_{k_{3}-1} c_{k_{3}}-r c_{k_{3}-1}-r c_{k_{3}}}{c_{1} c_{3} \ldots c_{k_{3}-1}} \leq \\
& \leq h\left(c_{1}, c_{3}, c_{4}, \ldots, c_{k_{3}}\right)+\frac{c_{1} c_{2}-r c_{1}-r c_{2}}{c_{1}}+\frac{c_{2} c_{3}-r c_{2}-r c_{3}}{c_{1}}-\frac{c_{1} c_{3}-r c_{1}-r c_{3}}{c_{1}} \\
& \leq h\left(c_{1}, c_{3}, c_{4}, \ldots, c_{k_{3}}\right)+\frac{\overbrace{\left(c_{1}-r\right)}^{\leq 0} c_{2}}{c_{1}}+\frac{c_{2} \cdot \overbrace{\left(c_{3}-r\right)}^{c_{1}}}{c_{1}}-\frac{c_{1} c_{3}}{c_{1}} \\
& \leq h\left(c_{1}, c_{3}, c_{4}, \ldots, c_{k_{3}}\right),
\end{aligned}
$$

which is a contradiction.

SUBCASE 1.2: $j \leq 2$.

If $k_{3}=2$, then $c_{2}=m$ and

$$
\begin{aligned}
0 & <-r c_{1}+\frac{c_{1} m-r c_{1}-r m}{c_{1}}< \\
& <-r c_{1}+\frac{c_{1} m-r c_{1}-r m}{c_{1}}+\frac{m^{2}-2 r m}{c_{1} m}+\frac{m^{2}-2 r m}{c_{1} m^{2}},
\end{aligned}
$$

hence $\left(c_{1}, m, m, m\right) \in S_{1}$. If $k_{3}=3$, then $c_{3}=m$ and

$$
\begin{aligned}
0 & <-r c_{1}+\frac{c_{1} c_{2}-r c_{1}-r c_{2}}{c_{1}}+\frac{c_{2} m-r c_{2}-r m}{c_{1} c_{2}}< \\
& <-r c_{1}+\frac{c_{1} c_{2}-r c_{1}-r c_{2}}{c_{1}}+\frac{c_{2} m-r c_{2}-r m}{c_{1} c_{2}}+\frac{m^{2}-2 r m}{c_{1} c_{2} m},
\end{aligned}
$$


hence $\left(c_{1}, c_{2}, m, m\right) \in S_{1}$. If $k_{3}>3$, then $c_{3} \geq r$ and all entries after $c_{3}$ are equal to $m$. Hence, in any case there is an element of $S_{1}$ of the form $c^{\prime}=(z_{1}, z_{2}, z_{3}, \underbrace{m \ldots, m}_{t \text {-times }})$, where $t \geq 1$ and $\left(z_{1}, z_{2}, z_{3}\right) \in X$. Since, $c^{\prime} \in S_{1}$, it follows that $f\left(c^{\prime}\right)>g\left(z_{1}, z_{2}, z_{3}\right)$, i.e.

$$
\begin{aligned}
& \frac{\frac{x_{1} x_{2}}{x_{1}}+\frac{x_{2} x_{3}}{x_{1} x_{2}}+\frac{x_{3} m}{x_{1} x_{2} x_{3}}+\frac{1}{x_{1} x_{2} x_{3}} \cdot m^{2} \cdot \frac{1-\frac{1}{m^{t}}}{m-1}}{x_{1}+\frac{x_{1}+x_{2}}{x_{1}}+\frac{x_{2}+x_{3}}{x_{1} x_{2}}+\frac{x_{3}+m}{x_{1} x_{2} x_{3}}+\frac{1}{x_{1} x_{2} x_{3}} \cdot 2 m \cdot \frac{1-\frac{1}{m^{t}}}{m-1}}> \\
& \frac{\frac{x_{1} x_{2}}{x_{1}}+\frac{x_{2} x_{3}}{x_{1} x_{2}}+\frac{x_{3} m}{x_{1} x_{2} x_{3}}+\frac{1}{x_{1} x_{2} x_{3}} \cdot m^{2} \cdot \frac{1}{m-1}}{x_{1}+\frac{x_{1}+x_{2}}{x_{1}}+\frac{x_{2}+x_{3}}{x_{1} x_{2}}+\frac{x_{3}+m}{x_{1} x_{2} x_{3}}+\frac{1}{x_{1} x_{2} x_{3}} \cdot 2 m \cdot \frac{1}{m-1}}
\end{aligned}
$$

Denote

$$
\begin{aligned}
\alpha_{1} & =\frac{x_{1} x_{2}}{x_{1}}+\frac{x_{2} x_{3}}{x_{1} x_{2}}+\frac{x_{3} m}{x_{1} x_{2} x_{3}} \\
\beta_{1} & =x_{1}+\frac{x_{1}+x_{2}}{x_{1}}+\frac{x_{2}+x_{3}}{x_{1} x_{2}}+\frac{x_{3}+m}{x_{1} x_{2} x_{3}} \\
\gamma_{1} & =\frac{1}{x_{1} x_{2} x_{3}} \cdot 2 m \cdot \frac{1-\frac{1}{m^{t}}}{m-1} \\
\delta_{1} & =\frac{1}{x_{1} x_{2} x_{3}} \cdot 2 m \cdot \frac{1}{m-1}
\end{aligned}
$$

Inequality (2.3) can be rewritten as

$$
\begin{aligned}
\frac{\alpha_{1}+\frac{m}{2} \gamma_{1}}{\beta_{1}+\gamma_{1}} & >\frac{\alpha_{1}+\frac{m}{2} \delta_{1}}{\beta_{1}+\delta_{1}} \\
\alpha_{1} \delta_{1}-\alpha_{1} \gamma_{1}+\frac{m}{2} \gamma_{1} \beta_{1}-\frac{m}{2} \beta_{1} \delta_{1} & >0 \\
\left(\alpha_{1}-\frac{m}{2} \beta_{1}\right)\left(\delta_{1}-\gamma_{1}\right) & >0
\end{aligned}
$$

Since $\delta_{1}>\gamma_{1}$,it follows that $\alpha_{1}-\frac{m}{2} \beta_{1}>0$, but

$$
\begin{aligned}
\alpha_{1}- & \frac{m}{2} \beta_{1}= \\
& =\frac{x_{1} x_{2}-\frac{m}{2} x_{1}-\frac{m}{2} x_{2}}{x_{1}}+\frac{x_{2} x_{3}-\frac{m}{2} x_{2}-\frac{m}{2} x_{3}}{x_{1} x_{2}}+\frac{x_{3} m-\frac{m}{2} x_{3}-\frac{m}{2} m}{x_{1} x_{2} x_{3}}-\frac{m}{2} x_{1} \\
& \leq\{\text { inequality between arithmetic and geometric mean }\} \leq \\
& \leq \frac{x_{1} x_{2}-m \sqrt{x_{1} x_{2}}}{x_{1}}+\frac{x_{2} x_{3}-m \sqrt{x_{2} x_{3}}}{x_{1} x_{2}}+\frac{x_{3} m-m \sqrt{x_{3} m}}{x_{1} x_{2} x_{3}}-\frac{m}{2} x_{1} \leq \\
& \leq\left\{m \geq \sqrt{x_{1} x_{2}}, \sqrt{x_{2} x_{3}}, \sqrt{x_{3} m}\right\} \leq-\frac{m}{2} x_{1} \leq 0
\end{aligned}
$$

which is a contradiction.

CASE 2: $h\left(c_{1}, \ldots, c_{k_{3}}\right)<h\left(c_{1}, c_{2}\right)$ 
In this case $h\left(c_{1}, c_{2}\right)>0$, hence $f\left(c_{1}, c_{2}\right)>\Psi(m) \geq g_{m}\left(c_{1}, c_{2}, m\right)$, i. e.

$$
\begin{aligned}
& \frac{\frac{c_{1} c_{2}}{c_{1}}}{c_{1}+\frac{c_{1}+c_{2}}{c_{1}}}>\frac{\frac{c_{1} c_{2}}{c_{1}}+\frac{c_{2} m}{c_{1} c_{2}}+\frac{m^{2}}{c_{1} c_{2} m}+\frac{1}{c_{1} c_{2} m} \cdot \frac{m^{2}}{m-1}}{c_{1}+\frac{c_{1}+c_{2}}{c_{1}}+\frac{c_{2}+m}{c_{1} c_{2}}+\frac{2 m}{c_{1} c_{2} m}+\frac{1}{c_{1} c_{2} m} \cdot \frac{2 m}{m-1}} \\
& \frac{c_{2}}{c_{1}+\frac{c_{1}+c_{2}}{c_{1}}}>\frac{c_{2}+\left(\frac{c_{2} m}{c_{1} c_{2}}+\frac{1}{c_{1} c_{2}} \cdot \frac{m^{2}}{m-1}\right)}{\left(c_{1}+\frac{c_{1}+c_{2}}{c_{1}}\right)+\left(\frac{c_{2}+m}{c_{1} c_{2}}+\frac{1}{c_{1} c_{2}} \cdot \frac{2 m}{m-1}\right)} .
\end{aligned}
$$

In order to obtain a contradiction, it is sufficient to prove that

$$
\begin{aligned}
\frac{\frac{c_{2} m}{c_{1} c_{2}}+\frac{1}{c_{1} c_{2}} \cdot \frac{m^{2}}{m-1}}{\frac{c_{2}+m}{c_{1} c_{2}}+\frac{1}{c_{1} c_{2}} \cdot \frac{2 m}{m-1}} & \geq \frac{c_{2}}{c_{1}+\frac{c_{1}+c_{2}}{c_{1}}} \\
\frac{c_{2} m+\frac{m^{2}}{m-1}}{c_{2}+m+\frac{2 m}{m-1}} & \geq \frac{c_{1} c_{2}}{c_{1}^{2}+c_{1}+c_{2}}
\end{aligned}
$$

Note that

$$
\frac{c_{1} c_{2}}{c_{1}^{2}+c_{1}+c_{2}} \leq \frac{c_{1} \cdot \frac{m}{2}+c_{2} \cdot \frac{m}{2}}{c_{1}^{2}+c_{1}+c_{2}} \leq \frac{m}{2}=\frac{\frac{m^{2}}{m-1}}{\frac{2 m}{m-1}},
$$

hence it is sufficient to prove that

$$
\frac{c_{2} m}{c_{2}+m} \geq \frac{c_{1} c_{2}}{c_{1}^{2}+c_{1}+c_{2}}
$$

This is equivalent to $c_{1}^{2} c_{2} m+c_{2}^{2} m \geq c_{1} c_{2}^{2}$,which obviously holds. Hence, a contradiction is obtained.

From Lemmas 2.7 and 2.11, our main result follows:

Theorem 2.12. Let $\Delta \geq 3$, then

$$
\Phi_{32}(\Delta)=2 \cdot \Psi(\Delta-1)
$$

This Theorem is very useful, because the number $\Psi(\Delta-1)$ can be determined in $\sim \Delta^{2}$ operations. The program for calculating the function $\Psi$ is produced and Table 1 of values for $\Phi_{32}(\Delta)$ is obtained.

Remark 2.13. Note that $X$ has $\sim \Delta^{2}$ elements. However, we can restrict our search for the maximum to only linear number of elements. Let $G_{m}:[1, m]^{3} \rightarrow R$ be the function defined by

$$
G_{m}\left(x_{1}, x_{2}, x_{3}\right)=\frac{\frac{x_{1} x_{2}}{x_{1}}+\frac{x_{2} x_{3}}{x_{1} x_{2}}+\frac{x_{3} m}{x_{1} x_{2} x_{3}}+\frac{1}{x_{1} x_{2} x_{3}} \cdot \frac{m^{2}}{m-1}}{x_{1}+\frac{x_{1}+x_{2}}{x_{1}}+\frac{x_{2}+x_{3}}{x_{1} x_{2}}+\frac{x_{3}+m}{x_{1} x_{2} x_{3}}+\frac{1}{x_{1} x_{2} x_{3}} \cdot \frac{2 m}{m-1}}
$$

Let

$$
h:[1, m] \rightarrow R
$$




\begin{tabular}{|c|c|c|c|c|c|c|c|c|c|}
\hline$\Delta$ & $\Phi_{32}(\Delta)$ & $\Delta$ & $\Phi_{32}(\Delta)$ & $\Delta$ & $\Phi_{32}(\Delta)$ & $\Delta$ & $\Phi_{32}(\Delta)$ & $\Delta$ & $\Phi_{32}(\Delta)$ \\
\hline 1 & Not def. & 41 & 6.6904 & 81 & 9.3623 & 121 & 11.3872 & 161 & 13.0876 \\
\hline 2 & 1.0000 & 42 & 6.7731 & 82 & 9.4180 & 122 & 11.4327 & 162 & 13.1281 \\
\hline 3 & 1.5000 & 43 & 6.8571 & 83 & 9.4730 & 123 & 11.4779 & 163 & 13.1683 \\
\hline 4 & 1.8750 & 44 & 6.9393 & 84 & 9.5273 & 124 & 11.5227 & 164 & 13.2083 \\
\hline 5 & 2.1538 & 45 & 7.0195 & 85 & 9.5810 & 125 & 11.5671 & 165 & 13.2480 \\
\hline 6 & 2.4074 & 46 & 7.0980 & 86 & 9.6339 & 126 & 11.6111 & 166 & 13.2875 \\
\hline 7 & 2.6667 & 47 & 7.1747 & 87 & 9.6862 & 127 & 11.6547 & 167 & 13.3267 \\
\hline 8 & 2.8913 & 48 & 7.2498 & 88 & 9.7379 & 128 & 11.6980 & 168 & 13.3657 \\
\hline 9 & 3.0877 & 49 & 7.3232 & 89 & 9.7889 & 129 & 11.7410 & 169 & 13.4045 \\
\hline 10 & 3.2609 & 50 & 7.3950 & 90 & 9.8442 & 130 & 11.7835 & 170 & 13.4430 \\
\hline 11 & 3.4146 & 51 & 7.4653 & 91 & 9.9000 & 131 & 11.8258 & 171 & 13.4813 \\
\hline 12 & 3.5794 & 52 & 7.5341 & 92 & 9.9552 & 132 & 11.8711 & 172 & 13.5193 \\
\hline 13 & 3.7500 & 53 & 7.6015 & 93 & 10.0098 & 133 & 11.9167 & 173 & 13.5571 \\
\hline 14 & 3.9080 & 54 & 7.6675 & 94 & 10.0638 & 134 & 11.9619 & 174 & 13.5947 \\
\hline 15 & 4.0546 & 55 & 7.7321 & 95 & 10.1172 & 135 & 12.0068 & 175 & 13.6321 \\
\hline 16 & 4.1912 & 56 & 7.8031 & 96 & 10.1701 & 136 & 12.0514 & 176 & 13.6692 \\
\hline 17 & 4.3186 & 57 & 7.8750 & 97 & 10.2224 & 137 & 12.0957 & 177 & 13.7062 \\
\hline 18 & 4.4378 & 58 & 7.9457 & 98 & 10.2741 & 138 & 12.1396 & 178 & 13.7429 \\
\hline 19 & 4.5495 & 59 & 8.0151 & 99 & 10.3253 & 139 & 12.1832 & 179 & 13.7793 \\
\hline 20 & 4.6730 & 60 & 8.0834 & 100 & 10.3760 & 140 & 12.2265 & 180 & 13.8156 \\
\hline 21 & 4.8000 & 61 & 8.1505 & 101 & 10.4261 & 141 & 12.2695 & 181 & 13.8516 \\
\hline 22 & 4.9211 & 62 & 8.2165 & 102 & 10.4757 & 142 & 12.3121 & 182 & 13.8900 \\
\hline 23 & 5.0367 & 63 & 8.2813 & 103 & 10.5248 & 143 & 12.3545 & 183 & 13.9286 \\
\hline 24 & 5.1472 & 64 & 8.3451 & 104 & 10.5734 & 144 & 12.3965 & 184 & 13.9669 \\
\hline 25 & 5.2528 & 65 & 8.4079 & 105 & 10.6215 & 145 & 12.4383 & 185 & 14.0050 \\
\hline 26 & 5.3540 & 66 & 8.4696 & 106 & 10.6691 & 146 & 12.4797 & 186 & 14.0430 \\
\hline 27 & 5.4509 & 67 & 8.5304 & 107 & 10.7162 & 147 & 12.5209 & 187 & 14.0807 \\
\hline 28 & 5.5439 & 68 & 8.5902 & 108 & 10.7629 & 148 & 12.5617 & 188 & 14.1182 \\
\hline 29 & 5.6331 & 69 & 8.6490 & 109 & 10.8091 & 149 & 12.6023 & 189 & 14.1555 \\
\hline 30 & 5.7322 & 70 & 8.7069 & 110 & 10.8589 & 150 & 12.6426 & 190 & 14.1927 \\
\hline 31 & 5.8333 & 71 & 8.7639 & 111 & 10.9091 & 151 & 12.6825 & 191 & 14.2296 \\
\hline 32 & 5.9313 & 72 & 8.8260 & 112 & 10.9588 & 152 & 12.7223 & 192 & 14.2663 \\
\hline 33 & 6.0262 & 73 & 8.8889 & 113 & 11.0081 & 153 & 12.7617 & 193 & 14.3028 \\
\hline 34 & 6.1182 & 74 & 8.9509 & 114 & 11.0570 & 154 & 12.8009 & 194 & 14.3392 \\
\hline 35 & 6.2073 & 75 & 9.0120 & 115 & 11.1054 & 155 & 12.8397 & 195 & 14.3753 \\
\hline 36 & 6.2939 & 76 & 9.0724 & 116 & 11.1534 & 156 & 12.8813 & 196 & 14.4112 \\
\hline 37 & 6.3778 & 77 & 9.1319 & 117 & 11.2010 & 157 & 12.9231 & 197 & 14.4470 \\
\hline 38 & 6.4594 & 78 & 9.1906 & 118 & 11.2481 & 158 & 12.9646 & 198 & 14.4826 \\
\hline 39 & 6.5386 & 79 & 9.2486 & 119 & 11.2949 & 159 & 13.0058 & 199 & 14.5180 \\
\hline 40 & 6.6156 & 80 & 9.3058 & 120 & 11.3412 & 160 & 13.0468 & 200 & 14.5532 \\
\hline
\end{tabular}

Figure 1: Table of values for for $\Phi_{32}(\Delta)$. 
be any function. Denote by MaxInt $(h)$ its maximum on the set $\{1, \ldots, m\}$. Let us observe the following functions

$$
\begin{gathered}
h_{m}:[1, m] \rightarrow R \text { defined by } h_{m}\left(x_{1}\right)=G_{m}\left(x_{1}, m, m\right) \\
\text { where } m \in N \backslash\{1\} ; \\
h_{x_{1}, m}:[1, m] \rightarrow R \text { defined by } h_{x_{1}, m}\left(x_{2}\right)=G_{m}\left(x_{1}, x_{2}, m\right) \\
\text { where } m \in N \backslash\{1\}, x_{1} \in N \text { and } x_{1} \leq m ; \\
h_{x_{1}, x_{2}, m}:[1, m] \rightarrow R \text { defined by } h_{x_{1}, x_{2}, m}\left(x_{3}\right)=G_{m}\left(x_{1}, x_{2}, x_{2}\right) \\
\text { where } m \in N \backslash\{1\}, x_{1}, x_{2} \in N \text { and } x_{1}, x_{2} \leq m .
\end{gathered}
$$

It can be easily seen that all of these functions are infinitely differentiable. Also, using Mathematica, it can be verified that they have at most two stationary points (null-points of the first derivation). Let $h:[1, m] \rightarrow R$ be an infinitely derivable function:

1) with no stationary points - then MaxInt $(h)=\max \{h(1), h(m)\}$;

2) with one stationary point $x$ - then MaxInt $(h)=\max \{h(1), h(m), h(\lfloor x\rfloor), h(\lceil x\rceil)\}$;

$3)$ with two stationary points $x$ and $y$ - then

$\operatorname{MaxInt}(h)=\max \{h(1), h(m), h(\lfloor x\rfloor), h(\lceil x\rceil), h(\lfloor y\rfloor), h(\lceil y\rceil)\}$.

Hence, in order to determine the $\Psi(m)$ it is sufficient to check at most

$$
6 \cdot\left(1+\left\lfloor\frac{m^{3 / 2}}{m+1}\right\rfloor+\left\lfloor\frac{m^{3 / 2}}{m+1}\right\rfloor^{2}\right)
$$

values, which can be done in linear time.

Remark 2.14. In order to prove that the function $\Phi_{32}$ is increasing, it is sufficient to show that

$$
\begin{aligned}
& \frac{\frac{x_{1} x_{2}}{x_{1}}+\frac{x_{2} x_{3}}{x_{1} x_{2}}+\frac{x_{3} m}{x_{1} x_{2} x_{3}}+\frac{1}{x_{1} x_{2} x_{3}} \cdot \frac{m^{2}}{m-1}}{x_{1}+\frac{x_{1}+x_{2}}{x_{1}}+\frac{x_{2}+x_{3}}{x_{1} x_{2}}+\frac{x_{3}+m}{x_{1} x_{2} x_{3}}+\frac{1}{x_{1} x_{2} x_{3}} \cdot \frac{2 m}{m-1}} \\
& \leq \frac{\frac{x_{1} x_{2}}{x_{1}}+\frac{x_{2} x_{3}}{x_{1} x_{2}}+\frac{x_{3}(m+1)}{x_{1} x_{2} x_{3}}+\frac{1}{x_{1} x_{2} x_{3}} \cdot \frac{(m+1)^{2}}{m}}{x_{1}+\frac{x_{1}+x_{2}}{x_{1}}+\frac{x_{2}+x_{3}}{x_{1} x_{2}}+\frac{x_{3}+m+1}{x_{1} x_{2} x_{3}}+\frac{1}{x_{1} x_{2} x_{3}} \cdot \frac{2(m+1)}{m}}
\end{aligned}
$$

for every $m \geq 3$ and $x_{1}, x_{2}, x_{3}$ which maximizes $g_{m}$. We need to prove that

$$
\begin{aligned}
& \frac{\frac{x_{1} x_{2}}{x_{1}}+\frac{x_{2} x_{3}}{x_{1} x_{2}}+\frac{x_{3} m}{x_{1} x_{2} x_{3}}+\frac{1}{x_{1} x_{2} x_{3}} \cdot \frac{m^{2}}{m-1}}{x_{1}+\frac{x_{1}+x_{2}}{x_{1}}+\frac{x_{2}+x_{3}}{x_{1} x_{2}}+\frac{x_{3}+m}{x_{1} x_{2} x_{3}}+\frac{1}{x_{1} x_{2} x_{3}} \cdot \frac{2 m}{m-1}} \\
&\left.\leq \frac{\left(\frac{x_{1} x_{2}}{x_{1}}+\frac{x_{2} x_{3}}{x_{1} x_{2}}+\frac{x_{3} m}{x_{1} x_{2} x_{3}}+\frac{1}{x_{1} x_{2} x_{3}} \cdot \frac{m^{2}}{m-1}\right)+}{\left(x_{1}+\frac{x_{1}+x_{2}}{x_{1}}+\frac{x_{2}+x_{3}}{x_{1} x_{2}}+\right.}+\frac{x_{3}+m}{x_{1} x_{2} x_{3}}+\frac{1}{x_{1} x_{2} x_{3}} \cdot \frac{2 m}{m-1}\right)+ \\
&+\frac{\left(\frac{x_{3}}{x_{1} x_{2} x_{3}}+\frac{1}{x_{1} x_{2} x_{3}} \cdot\left(\frac{(m+1)^{2}}{m}-\frac{m^{2}}{m-1}\right)\right)}{} \\
&+\left(\frac{1}{x_{1} x_{2} x_{3}}+\frac{1}{x_{1} x_{2} x_{3}} \cdot\left(\frac{2(m+1)}{m}-\frac{2 m}{m-1}\right)\right)
\end{aligned}
$$


It is sufficient to show that

$$
\begin{aligned}
& \frac{\frac{x_{1} x_{2}}{x_{1}}+\frac{x_{2} x_{3}}{x_{1} x_{2}}+\frac{x_{3} m}{x_{1} x_{2} x_{3}}+\frac{1}{x_{1} x_{2} x_{3}} \cdot \frac{m^{2}}{m-1}}{x_{1}+\frac{x_{1}+x_{2}}{x_{1}}+\frac{x_{2}+x_{3}}{x_{1} x_{2}}+\frac{x_{3}+m}{x_{1} x_{2} x_{3}}+\frac{1}{x_{1} x_{2} x_{3}} \cdot \frac{2 m}{m-1}} \leq \frac{\frac{x_{3}}{x_{1} x_{2} x_{3}}+\frac{1}{x_{1} x_{2} x_{3}} \cdot\left(\frac{(m+1)^{2}}{m}-\frac{m^{2}}{m-1}\right)}{\frac{1}{x_{1} x_{2} x_{3}}+\frac{1}{x_{1} x_{2} x_{3}} \cdot\left(\frac{2(m+1)}{m}-\frac{2 m}{m-1}\right)} \\
& \frac{\frac{x_{1} x_{2}}{x_{1}}+\frac{x_{2} x_{3}}{x_{1} x_{2}}+\frac{x_{3} m}{x_{1} x_{2} x_{3}}+\frac{1}{x_{1} x_{2} x_{3}} \cdot \frac{m^{2}}{m-1}}{x_{1}+\frac{x_{1}+x_{2}}{x_{1}}+\frac{x_{2}+x_{3}}{x_{1} x_{2}}+\frac{x_{3}+m}{x_{1} x_{2} x_{3}}+\frac{1}{x_{1} x_{2} x_{3}} \cdot \frac{2 m}{m-1}} \leq \frac{\left(m^{2}-m\right) x_{3}+m^{2}-m-1}{\left(m^{2}-m\right)-2} .
\end{aligned}
$$

From the proof of the Lemma 2.11, it follows that

$$
\frac{\frac{x_{1} x_{2}}{x_{1}}+\frac{x_{2} x_{3}}{x_{1} x_{2}}+\frac{x_{3} m}{x_{1} x_{2} x_{3}}+\frac{1}{x_{1} x_{2} x_{3}} \cdot \frac{m^{2}}{m-1}}{x_{1}+\frac{x_{1}+x_{2}}{x_{1}}+\frac{x_{2}+x_{3}}{x_{1} x_{2}}+\frac{x_{3}+m}{x_{1} x_{2} x_{3}}+\frac{1}{x_{1} x_{2} x_{3}} \cdot \frac{2 m}{m-1}} \leq x_{3}
$$

and the claim follows.

The behavior of this function on its boundary is described by the following theorem:

Theorem 2.15. It holds that

$$
\lim _{\Delta \rightarrow \infty} \frac{\log \Phi_{32}(\Delta)}{\log \Delta}=\frac{1}{2} .
$$

Proof. First, let us prove that $\lim _{\Delta \rightarrow \infty} \frac{\log \Phi_{32}(\Delta)}{\log \Delta} \leq \frac{1}{2}$. We have:

$$
\begin{aligned}
\lim _{\Delta \rightarrow \infty} \frac{\log \Phi_{32}(\Delta)}{\log \Delta} & =\lim _{\Delta \rightarrow \infty} \frac{\log (2 \cdot \Gamma(\Delta-1))}{\log \Delta} \leq\{\text { from Lemma } 2.8\} \leq \\
& \leq \lim _{\Delta \rightarrow \infty} \frac{\log \left(2 \cdot \frac{(\Delta-1)^{3 / 2}}{\Delta}\right)}{\log \Delta} \leq \lim _{\Delta \rightarrow \infty}\left(\frac{\log 2+\frac{1}{2} \log \Delta}{\log \Delta}\right)=\frac{1}{2} .
\end{aligned}
$$

Now, let us prove that $\lim _{\Delta \rightarrow \infty} \frac{\log \Phi_{32}(\Delta)}{\log \Delta} \geq \frac{1}{2}$. We have:

$$
\begin{aligned}
& \lim _{\Delta \rightarrow \infty} \frac{\log \Phi_{32}(\Delta)}{\log \Delta}=\lim _{\Delta \rightarrow \infty} \frac{\log (2 \cdot \Gamma(\Delta-1))}{\log \Delta} \geq \lim _{\Delta \rightarrow \infty} \frac{\log (2 \cdot \Psi(\Delta-1))}{\log \Delta} \\
& \geq \lim _{\Delta \rightarrow \infty} \frac{\log \left(2 \cdot g_{\Delta-1}(\lceil\sqrt{\Delta-1\rceil, \Delta, \Delta))}\right.}{\log \Delta} \\
& =\lim _{\Delta \rightarrow \infty} \frac{\log \left(2 \cdot \frac{\mid \sqrt{\Delta-1}\rceil \cdot \Delta-1}{\lceil\sqrt{\Delta-1}\rceil}+\frac{\Delta-1}{\lceil\sqrt{\Delta-1}\rceil}+\frac{1}{\lceil\sqrt{\Delta-1}\rceil}+\frac{1}{\lceil\sqrt{\Delta-1}\rceil \cdot(\Delta-2)}\right.}{\left.\frac{\mid \sqrt{\Delta-1}\rceil+\frac{\sqrt{\Delta-1}\rceil+\Delta-1}{\lceil\sqrt{\Delta-1}\rceil}+\frac{2}{\lceil\sqrt{\Delta-1}\rceil}+\frac{2}{\lceil\sqrt{\Delta-1}\rceil \cdot(\Delta-1)}+\frac{2}{\lceil\sqrt{\Delta-1}\rceil \cdot(\Delta-1) \cdot(\Delta-1)}}{\log \Delta}\right)} \\
& \geq \lim _{\Delta \rightarrow \infty} \frac{\log \left(2 \cdot \frac{\Delta-1}{9\lceil\sqrt{\Delta-1}\rceil}\right)}{\log \Delta} \geq \lim _{\Delta \rightarrow \infty} \frac{\log \left(\frac{2}{9} \cdot \sqrt{\Delta-1}\right)}{\log \Delta}=\frac{1}{2} .
\end{aligned}
$$

\section{Acknowledgement}

Partial support of Croatian Ministry of Science, Education and Sports is gratefully acknowledged (grant no. 177-0000000-0884 and grant no. 037-0000000-2779). Help with English language of Sarah Michelle Rajtmajer is also gratefully acknowledged. 


\section{References}

[1] M. Aouchiche, J. M. Bonnefoy, A. Fidahoussen, G. Caporossi, P. Hansen, P. L. Hiesse, J. Lacheré and A. Monhait, Variable neighborhood search for extremal graphs 14, The AutoGraphiX 2 system, in: L. Liberti and N. Maculan (eds.), Global Optimization: From Theory to Implementation, Springer, 2005.

[2] B. Bollobás, Extremal Graph Theory, Academic Press, London, 1978.

[3] I. Gutman and K. C. Das, The First Zagreb Index 30 Years After, MATCH - Commun. Math. Comput. Chem. 50 (2004), 83-92.

[4] P. Hansen and D. Vukičević, Comparing the Zagreb Indices, Croatica Chemica Acta 80 (2007), $165-168$.

[5] W. Imrich, Explicit Construction of Regular Graphs Without Small Cycles, Combinatorica 4 (1984), 53-39.

[6] S. Nikolić, G. Kovačević, A. Miličević and N. Trinajstić, The Zagreb Indices 30 Years After, Croat. Chem. Acta 76 (2003), 113-124.

[7] R. Todeschini and V. Consonni, Handbook of Molecular Descriptors, Wiley-VCH, Weinheim, 2000.

[8] N. Trinajstić, Chemical Graph Theory, CRC Press, Boca Raton, 1992.

[9] D. Vukičević, Comparing variable Zagreb indices, MATCH - Commun. Math. Comput. Chem. 57 (2007), 633-641.

[10] D. Vukičević and A. Graovac, Comparing Zagreb M1 and M2 indices for acyclic molecules, MATCH - Commun. Math. Comput. Chem. 57 (2007), 587-590.

[11] D. Vukičević and A. Graovac, Comparing variable Zagreb M1 and M2 indices for acyclic molecules, MATCH - Commun. Math. Comput. Chem. 60 (2008), 37-44. 